\title{
Absolventenbefragung des Instituts für Statistik der Technischen Universität Graz
}

\author{
Ernst Stadlober, Erwin Stampfer \\ Institut für Statistik, Technische Universität Graz
}

\begin{abstract}
Zusammenfassung. Im Rahmen der 25-Jahr-Feier des Instituts für Statistik der TU Graz wurde eine Umfrage unter den Absolventinnen und Absolventen des Instituts zum Thema Studium und Beruf durchgeführt. Dieser Artikel faßt in aller Kürze die wichtigsten Ergebnisse dieser Umfrage zusammen.
\end{abstract}

Abstract. On celebrating its $25^{\text {th }}$ anniversary the Institute of Statistics at the Graz University of Technology conducted a survey among its graduates. This article contains a condensed summary of its most important results.

Schlüsselwörter: Abolventenbefragung, survey among graduates.

\section{Einleitung}

Anläßlich der 25-Jahr-Feier des Instituts für Statistik an der Technischen Universität Graz im April 1998 wurden Fragebögen an alle Absolventen ${ }^{1}$, welche an diesem Institut ihre Diplomarbeit (bzw. Dissertation) geschrieben hatten, versandt. Das Ziel war es, aussagekräftige Informationen zum Studium, zum derzeitigen Beruf, zur Relevanz des Studiums für die berufliche Praxis und zur aktuellen Stellensituation zu sammeln und statistisch auszuwerten.

Die Erhebung umfaßte ein Kollektiv von Absolventen des Studiums der Technischen Mathematik. Es setzte sich aus allen Studenten (insgesamt 110) zusammen, welche zwischen 1975 und 1997 ihre Diplomarbeit am Institut für Statistik angefertigt hatten.

Beinahe zwei Drittel der Befragten (69 von 110) hatten den Fragebogen ausgefüllt und zurückgeschickt. Durch die hohe Rücklaufrate und einer Aufteilung der Rücksendungen bzgl. der Kategorien Geschlecht und Studienzweig, die ungefähr jener in der Gesamtpopulation entsprach, sind die folgenden Aussagen als repräsentativ für Absolventen des Instituts für Statistik der TU Graz anzusehen.

Von den Befragten hatten 22 (31\%) das Studium der Technischen Mathematik vor 1988 abgeschlossen und 47 (69\%) nach 1988. 28 (41\%) haben den Studienzweig B (Wirtschafts- und Planungsmathematik, der erst seit 1985 an der TU-Graz eingerichtet ist, mit Absolventen ab 1991), 41 (59\%) den Studienzweig C (Informations- und Datenverarbeitung) gewählt. Unter den Befragten war keiner, der den Studienzweig A (Naturwissenschaften) absolviert hat. 14 der 69 Befragten waren weiblichen Geschlechts, was einem Anteil von 20\% weiblicher Absolventen entspricht.

\footnotetext{
${ }^{1}$ Hier und im folgenden sind Hinweise auf Personen (z.B. Absolvent, Student) als geschlechtsneutral zu verstehen.
} 
Der vollständige Bericht inklusive Fragebogen ist per Internet unter

http://www.tu-graz.ac.at/stat/archiv/befragung.doc

erhältlich.

\section{Tätigkeitsbereiche und Branchen}

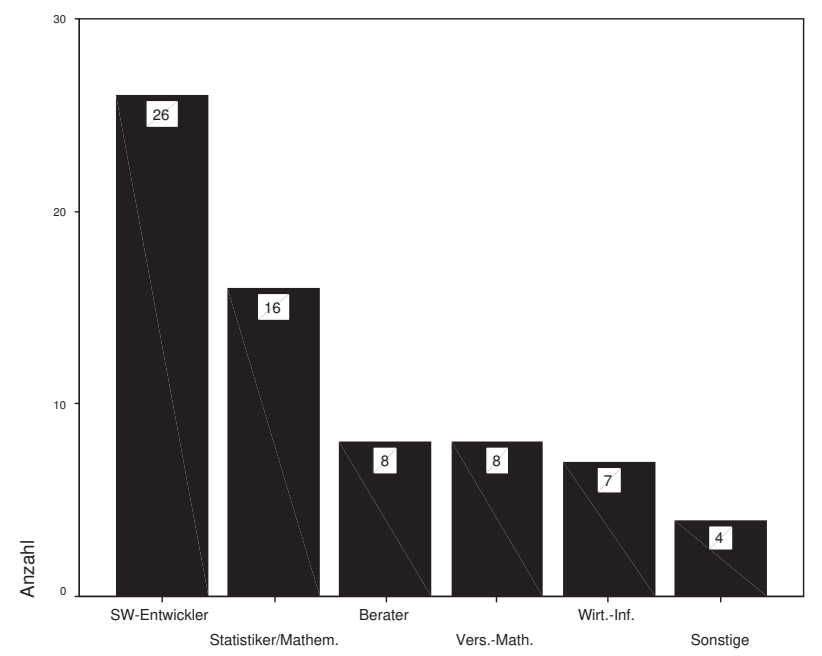

Abbildung 1: Tätigkeitsbereich der Absolventen.

Am häufigsten sind Absolventen (besonders jene, die den Zweig C: Informations- und Datenverarbeitung gewählt haben) mit Entwickeln von Software (38\%) beschäftigt. Ein nicht unbeträchtlicher Anteil ist als Mathematiker-Statistiker (23\%), ein etwas kleinerer Anteil als Versicherungsmathematiker und Berater (jeweils 12\%) sowie als Wirtschaftsinformatiker (10\%) tätig. Der kleine Rest splittert sich in sonstige Tätigkeitsbereiche auf (siehe Abbildung 1).

Die Branchen, in denen die Technischen Mathematiker vornehmlich tätig wurden, sind die Softwarebranche (28\%), Forschung \& Entwicklung (20\%), Versicherungen und Banken (12\%), öffentliche Körperschaften (12\%) und andere (siehe Abbildung 2).

\section{Einkommen und regionale Verteilung}

Erfreulicherweise würden 72 Prozent der Absolventen - vor die Wahl gestellt - das Studium der Technischen Mathematik einem anderen Studium oder einem Fachhochschullehrgang vorziehen, zumal die Einkommensaussichten als Angestellter im mittleren Management $(20 \%)$ oder als Führungskraft (12\%) äußerst gut sind. 16 Prozent gaben ein Jahresbruttoeinkommen von mehr als ATS 700.000 an. Weitere 16 Prozent scheuten sich aber, Angaben über ihr Einkommen zu machen. Die Mehrheit der Befragten (65\%) lag mit dem Jahresbruttoeinkommen zwischen ATS 300.000 und ATS 700.000. 


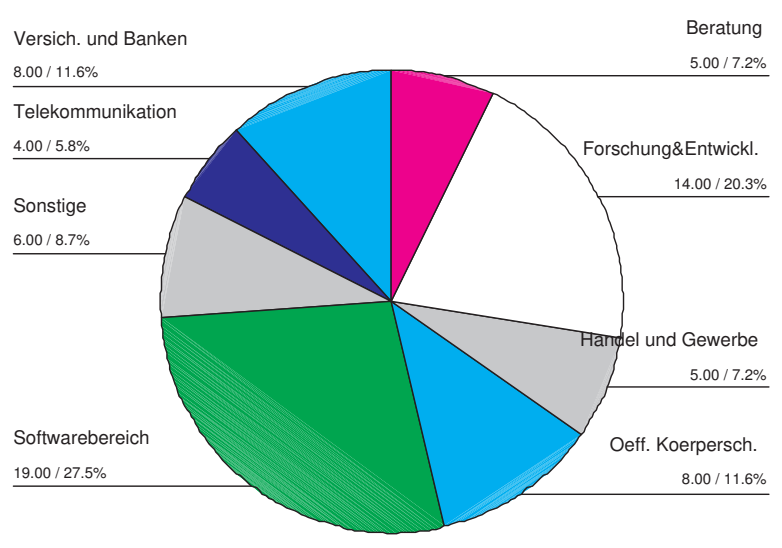

Abbildung 2: Branchen, in welchen die Absolventen tätig sind.

Die Befragung hat weiters ergeben, daß 61\% der Absolventen im Großraum Graz eine Anstellung gefunden hat; der Rest verteilt sich auf den Großraum Wien (15\%), Europa $(12 \%)$, Kärnten (10\%) und auf die übrigen Bundesländer.

\section{Studiendauer}

Bezüglich der durchschnittlichen Studiendauer ergaben sich einerseits keine signifikanten Unterschiede zwischen den weiblichen und männlichen Absolventen, und andererseits keine signifikanten Unterschiede zwischen den Studienzweigen B und C. Der ermittelte Wert von ca. 12,5 Semester liegt deutlich unter der durchschnittlichen Studiendauer anderer Studienrichtungen an der Technischen Universität Graz.

\section{Relevanz des Studiums}

52 Prozent der Befragten maßen dem Studium der Technischen Mathematik große, aber nur 10 Prozent geringe Bedeutung für ihre berufliche Tätigkeit bei. Allerdings gibt es einige Wissensbereiche und allgemeine Fähigkeiten, über die die Befragten im Laufe ihres Studiums gerne mehr erfahren hätten.

An erster Stelle ist hier das Erwerben von kommunikativer Kompetenz zu nennen, die 61 Prozent der Absolventen während ihres Studiums vermißt hatten. Dazu zählen in erster Linie sprachliche Fähigkeiten und Präsentationstechniken.

Ein weiteres großes Manko stellte der Bereich der sozialen Kompetenz, wie Mitarbeiter- und Teamführung (30\% der Befragten) dar. Auffallend dabei ist, daß die männlichen Absolventen einen signifikant höheren Bedarf nach sozialer Kompetenz anmeldeten als die weiblichen. 
Als dritter Mangel wird den Absolventen zufolge die zum Teil fehlende wirtschaftliche Ausbildung angesehen (29\%). Wissensbedarf bestand unter anderem in den Bereichen Controlling, Marketing und Kostenrechnung. Dies wurde insbesondere von den Absolventen des Studienzweigs C (Informations- und Datenverarbeitung) moniert. Dazu ist zu erwähnen, daß für Absolventen des Zweiges B (Wirtschafts- und Planungsmathematik) der Bedarf durch Pflichtfächer aus den oben genannten Bereichen offensichtlich gut genug abgedeckt war. Außerdem stieg der Wunsch nach einer besseren Wirtschaftsausbildung mit der Position im Unternehmen. So verlangten nur 6 von 30 Sachbearbeitern geeignete Wirtschaftsfächer, während 6 von 8 Führungskräften eine bessere Wirtschaftsausbildung forderten.

Des weiteren hielten 13 Prozent der Befragten ein breiter gestreutes bzw. ein qualitativ besseres Informatikwissen für notwendig. Je nach belegtem Studienzweig wurde Bedarf nach tieferen Kenntnissen im Umgang mit Anwendersoftware oder mit verschiedenen Programmiersprachen angemeldet. Bemerkenswert dabei ist, daß Absolventinnen einen signifikant höheren Bedarf an einer fundierteren EDV-Ausbildung hatten als ihre männlichen Kollegen.

\section{Zusatzqualifikationen}

Viele Absolventen haben die in Abschnitt 5 erwähnten Wissensdefizite durch zusätzliche, zum Teil außeruniversitäre Aktivitäten wettgemacht. Ca. 14 Prozent haben nach dem Diplom noch das Doktorat erworben. Rund 38 Prozent gaben an, Sprachkurse (Fremdsprachen-, Rhetorikkurse) absolviert zu haben. 23 Prozent hatten sogar einen Auslandsaufenthalt zu verbuchen. Die Kenntnisse in (Betriebs-)Wirtschaft und in EDV wurden von 28 bzw. 32 Prozent der Befragten durch den Besuch von Kursen, Seminaren oder Vorlesungen verbessert.

\section{Einschätzen der aktuellen Stellensituation}

Ca. 60 Prozent der Absolventen konnten innerhalb eines Monats eine Stelle finden. Nach 6 Monaten hatten praktisch alle (bis auf 2) den Einstieg in das Berufsleben geschafft (siehe Abbildung 3).

Mehr als zwei Drittel der Absolventen meinten, daß es genügend Stellen gäbe, die mit Technischen Mathematikern besetzt werden könnten; vor allem Softwareentwickler, Statistiker/Mathematiker und Versicherungsmathematiker vertraten diese Ansicht. Insgesamt würden knapp 80 Prozent der Befragten die Einstellung eines Technischen Mathematikers in ihrer Firma befürworten, was die positive Einschätzung der Stellensituation noch verstärkte. 


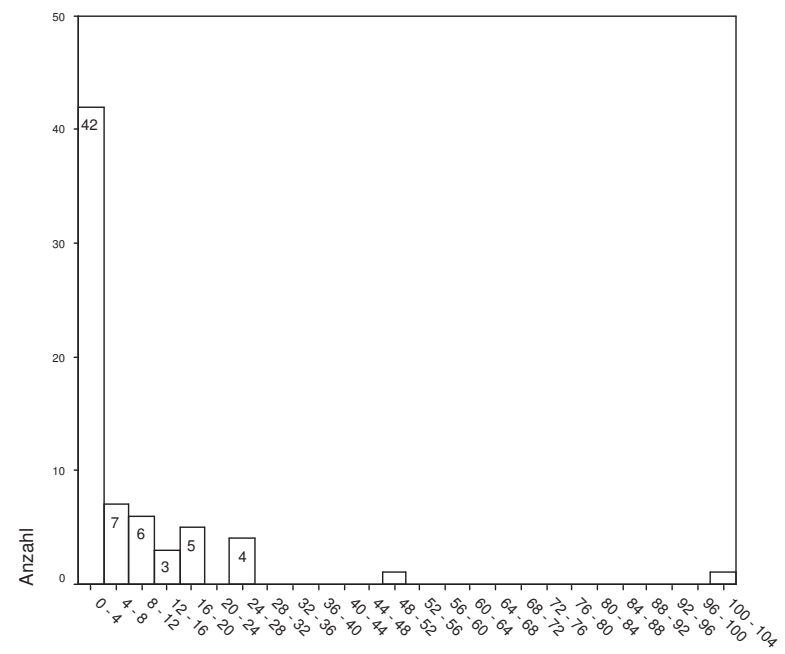

Abbildung 3: Dauer der Stellensuche (in Wochen).

Adresse der Autoren:

Univ.-Prof. Dipl.-Ing. Dr. Ernst Stadlober Univ.-Ass. Dipl.-Ing. Erwin Stampfer

Institut für Statistik

Technische Universität Graz

Steyrergasse 17/IV

$8020 \mathrm{Graz}$

Tel.: +43316873/6478

E-mail: stadlober@stat.tu-graz.ac.at Web: http://www.tu-graz.ac.at/stat/ 